ScIDoC

International Journal of Dentistry and Oral Science (IJDOS)

ISSN: $2377-8075$

\title{
Clinical Relevance Of RDC Diagnostic Tool In Current Clinical Environment: Temporomandibular Disorder Study - A Point Pandect
}

Research Article

\section{Vaishnavi Rajaraman ${ }^{1 *}$, Deepak Nallaswamy ${ }^{2}$, Subha $\mathrm{M}^{3}$}

${ }^{1}$ Post Graduate, Department of Prosthodontics and Implantology, Saveetha Dental College and Hospital,Saveetha Institute Of Medical And Technical Sciences, Saveetha University, Chennai, India.

${ }^{2}$ Professor, Department of Prosthodontics and Implantolgy, Saveetha Dental College and Hospital, Saveetha Institute Of Medical And Technical Sciences, Saveetha University, Chennai, India.

${ }^{3}$ Associate Professor, Department of Oral Medicine \& Radiology, Saveetha Dental College and Hospital, Saveetha Institute Of Medical And Technical Sciences,Saveetha University, Chennai, India.

Abstract

Background: Temporomandibular Disorders(TMD) are associated with the Temporomandibular Joint (TMJ) that can cause pain in the joint and in the muscles that control jaw movement. Patient evaluation, with various imaging modalities, as adjuvant, may help to interpret a patient's stage of TMD, aiding in diagnosis and treatment planning. Only a small percentage of afflicted individuals , affected with TMD, seek treatment, while up to $25 \%$ of the population may experience symptoms.

Owing to the diverse nature of TMD symptoms, physical examination along with various imaging modalities are often essential for patient evaluation.As result, TMD identification may involve any combination of the following modalities: a thorough history, physical examination, plain and panoramic radiography, arthrography, conventional and computed tomography (CT), MRI.

RDC/TMD was published by a group of American researchers in 1992. This protocol aimed to :

a. Critically revise existing TMD diagnostic systems in use

b. Critically revise the reliability and validity of existingTMD examination methods

c. Standardise TMD examination procedure

d. Establish research diagnostic criteria for TMD

e. Assess pain related disability and psychological status in TMD patients.

Aim: To assess the knowledge and awareness among dental practitioners about the Research Diagnostic Criteria for Temporomandibular Disorders (RDC/TMD).

Materials and Methods: A well-structured online multiple choice questionnaire was formulated with 5 questions on the RDC/ TMD. The sample size was chosen by simple randomised sampling as 100 dentists in Chennai, practicing general dentistry, irrespective of their speciality. The questionnaire was circulated to 100 participants including, postgraduate students, staffs in institutions, private practitioners and results were tabulated.

Results: Among the participants of the survey conducted, $82.1 \%$ were female and $17.9 \%$ were male dental clinicians. The data acquired from this survey exhibited that $72.7 \%$ of dentists were aware about the RDC/TMD. Most dentists knew the bimanual technique of palpation of TMJ (88.1\%). Dentists who come across cases of TMD are $68.7 \%$.Among the population studied, $75.4 \%$ dentists did not use RDC/TMD for diagnosis of TMD. If TMD was suspected, $88.1 \%$ dentists referred the patient for a thorough radiographic investigation. The data of this survey was compiled and represented graphically.

Conclusion: The majority of the patients would report symptoms that are already identified as risk factors for developing TMD and pain conditions. Diagnosis of TMD at this stage and correct identification of these symptoms is essential. Although this tool is the 'gold standard', The Research Diagnostic Criteria for TMD (RDC/TMD) is rarely used in general practice. Therefore it is necessary for all dental clinicians to be aware of TMD symptoms

Keywords: Research Diagnostic Criteria for Temporomandibular Disorders (RDC/TMD); Temporomandibular Disorders (TMD); Temporomandibular Joint (TMJ); Diagnosis; Awareness.

*Corresponding Author:

Vaishnavi Rajaraman,

Post-Graduate, Department of Prosthodontics and Implantology, Saveetha Dental College and Hospital,Saveetha Institute Of Medical And Technical Sciences, Saveetha University, Chennai, India.

Tel: 8779565950

E-mail: drvaish.sav@gmail.com

Received: June 17, 2020

Accepted: September 10, 2020

Published: October 07, 2020

Citation: Vaishnavi Rajaraman, Deepak Nallaswamy, Subha M. Clinical Relevance Of RDC Diagnostic Tool In Current Clinical Environment: Temporomandibular Disorder Study A Point Pandect. Int J Dentistry Oral Sci. 2020;7(10):844-848. doi: http://dx.doi.org/10.19070/2377-8075-20000166

Copyright: Vaishnavi Rajaraman 2020. This is an open-access article distributed under the terms of the Creative Commons Attribution License, which permits unrestricted use, distribution and reproduction in any medium, provided the original author and source are credited. 


\section{Introduction}

TMJ disorders (TMD) are a class of degenerative musculoskeletal conditions associated with morphological and functional deformities [1-5]. TMD include abnormalities of the intra-articular discal position and/or structural dysfunction of the associated musculature [6-10]. Symptoms and signs include painful joint sounds, restricted or deviating range of motion, and cranial and/or muscular pain known as orofacial pain [11-14]. Due to the complex and unique nature of every TMD case encountered, its diagnosis requires patient-specific analysis which should be accompanied by various diagnostic modalities.

Since the American Dental Association's president's conference on temporomandibular disorders (TMDs) in 1983, every major forum on this topic has highlighted the need for a reliable and valid diagnostic classification system to identify TMD cases, including specific subtypes [15]. In particular, the National Institutes of Health Technology Assessment Conference Statement on the Management of Temporomandibular Disorders released in 1996 articulated the need for epidemiological and experimental studies to determine the etiologic mechanisms of and risk factors for TMDs [16]. Results from such studies would provide the basis for an etiology-based diagnostic classification system necessary to best facilitate clinical research leading to improved management and treatments for these disorders [11-14].

The Research Diagnostic Criteria for Temporomandibular Disorders (RDC/TMD) are a widely employed diagnostic protocol for TMD research [7]. This taxonomic system includes an Axis I physical assessment and diagnostic protocol and an Axis II assessment of psychological status and pain-related disability [7, 17]. The (RDC/TMD) presented by Dworkin and LeResche in 1992 provided an important first step towards an etiology-based system. This symptom-based system provides well-defined operational definitions to distinguish TMD cases from controls, as well as to diagnose specific TMD subtypes.

The RDC/TMD has been used in many epidemiologic and clinical studies of TMDs. The aim of the present study was to assess the knowledge and awareness among dental practitioners about the RDC/TMD.

\section{Materials and Methods}

The Research Diagnostic Criteria (RDC) categorise TMD criteria into 3 groups according to the common factors among conditions. TMD's RDC groups are:

GROUP I: Muscle disorders:
I.a Myofascial pain

I.b Myofascial pain with limited opening

GROUP II: Disc Displacements (DD):

II.a DD with reduction

II.b DD without reduction with limited opening

II.c DD without reduction without limited opening

GROUP III: Other common Joint disorders:

III.a Arthralgia

III.b Osteoarthritis

III.c Osteoarthrosis

A subject can be allocated a maximum of one muscle disorder. In addition, only one diagnosis from each group (II and III) can be assigned to each joint. Therefore, at one time, a number of diagnoses can be given to one person widely ranging from 0 (no TMD diagnosis) up to 5 . However, to assign more than 3 diagnoses to one patient,is uncommon [18]. A well-structured multiple choice based structured questionnaire was framed. It was circulated, by electronic media, to the dentists involved in clinical practice.

The sample size was chosen by simple randomised sampling as 100 clinicians practicing in Chennai, from the data obtained on all current practitioners in the city. Demographic data of gender and the designation of the dental clinicians were taken. Personal information was not taken to avoid bias in the survey.

The questionnaire was circulated to around 150 clinicians and 100 participants answered the survey. The questionnaire was circulated to all participants including, postgraduate students, staffs in institutions, private practitioners and results were tabulated. A total of 5 questions were formulated based on the awareness of RDC/TMD for diagnosis of TMD [Tabe 1].

\section{Results}

Among the participants of the survey conducted, $82.1 \%$ were female and $17.9 \%$ were male dental clinicians. The participants of survey included $22.7 \%$ practitioners in private clinic, $50 \%$ postgraduate students, $18.2 \%$ students in their internship, and $9.1 \%$ undergraduate students [Figure 2]. The data acquired from this survey exhibited that $72.7 \%$ of dentists were aware, while $27.3 \%$ were unfamiliar about the RDC/TMD [Figure 3]. Around $88.1 \%$ dentists were familiar with the bimanual technique of palpation of TMJ while $11.9 \%$ of them were unaware about the same [Figure 4]. Among the participants of this survey, $68.7 \%$ dentists encountered cases with TMD and 31.3\% did not come across such

Table 1. Table showing the questionnaire included in the survey.

\begin{tabular}{|c|c|}
\hline S.NO & SURVEY QUESTION \\
\hline 1 & ARE YOU AWARE ABOUT THE RDC CRITERIA ?? \\
\hline 2 & ARE YOU AWARE ABOUT THE BIMANUAL PALPATION TECHNIQUE ?? \\
\hline 3 & DO YOU ENCOUNTER TMD CASES IN YOUR CLINIC ?? \\
\hline 4 & DO YOU USE RDC FOR TMD DIAGNOSIS ?? \\
\hline 5 & DO YOU SEND THE SUSPECTED PATIENTS FOR RADIOGRAPHIC INVESTIGATION ?? \\
\hline
\end{tabular}


Figure 1. Designation of the participants of the survey.

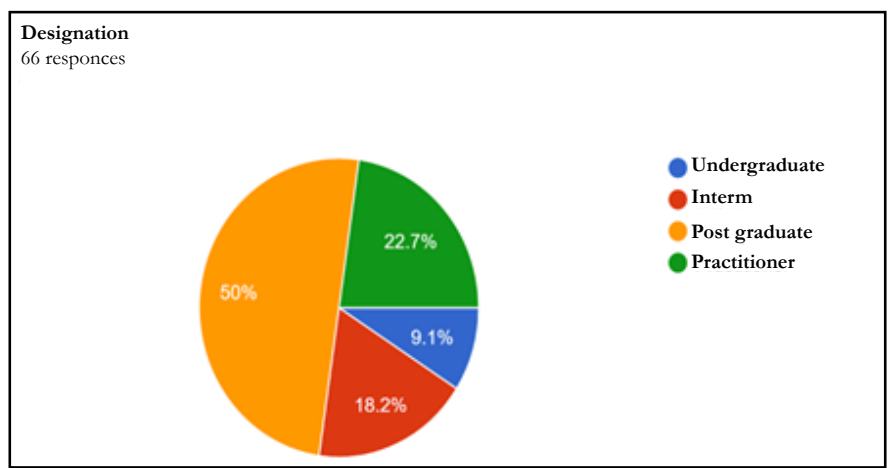

Figure 2. Awareness about the RDC criteria.

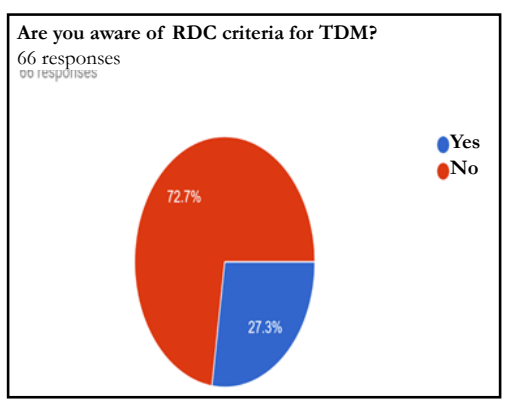

Figure 3. Awareness about the bimanual palpation technique.

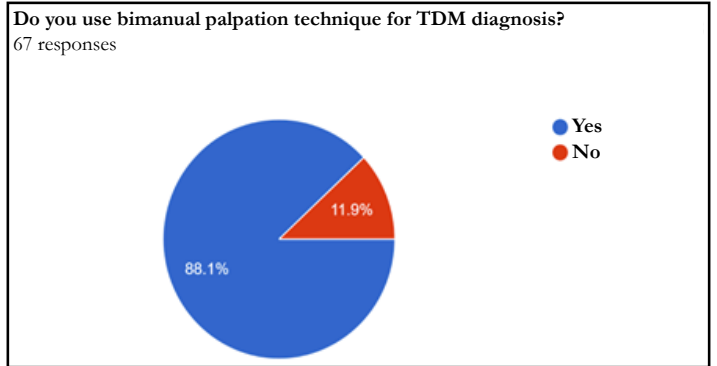

Figure 4. Number of TMD cases in the clinic.

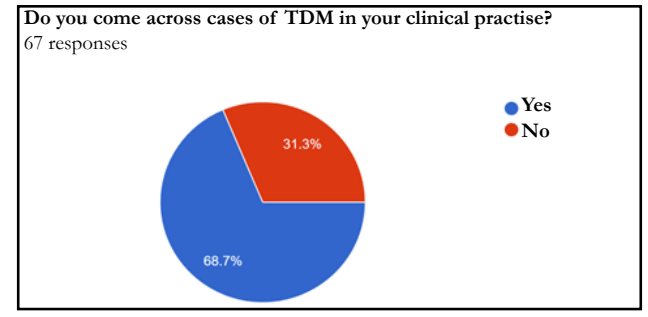

Figure 5. Using RDC for TMD diagnosis.

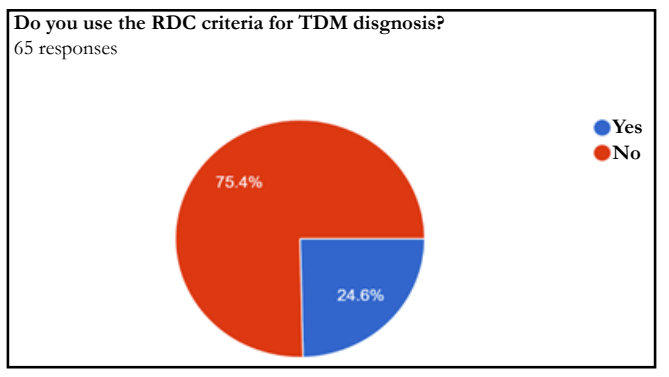

cases in routine practice [Figure 5]. Ony $24.6 \%$ the population studied, used the RDC/TMD for diagnostic criteria to suspect any TMD, while the rest $75.4 \%$ did not [Figure 6]. If TMD was suspected, $88.1 \%$ of the participants studied, referred the patient for a thorough radiographic investigation whereas $11.9 \%$ failed to refer [Figure 7].

\section{Discussion}

This survey highlights the awareness of dental clinicians about 
Figure 6. Suspected patients sent for radiographic investigation.

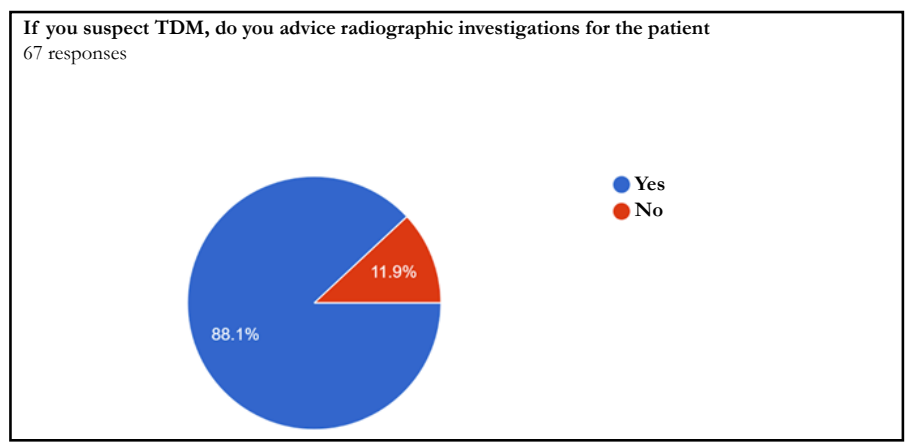

TMD and its diagnosis in specific.Among the population studied, $75.4 \%$ dentists did not use RDC/TMD for diagnosis of TMD. Although this tool is the 'gold standard', the Research Diagnostic Criteria for TMD (RDC/TMD) is rarely used in general practice [19]. The results of this survey emphasises the need for enhancing awareness among dental clinicians regarding the use of protocols for diagnosis for TMD.

Despite the existence of these diagnostic criteria, many general dental practitioners tend to look upon TMD diagnosis and management with a large degree of confusion and ambiguity. Tegelberg et al., [5] found that many dentists lacked routines for making diagnoses and only $25-50 \%$ of dentists reported positive experiences in relation to TMD diagnosis and management [20]. Another study found that a fear of misdiagnosing TMD, was common among general dental practitioners and hence often these patients were referred to specialist centres or hospital setting [21].

A team of researchers at Newcastle created a new diagnostic protocol for TMD7 which has been in clinical use for over 5 years; this is referred to as the clinical examination protocol-TMD (CEP-TMD) [22].

To simplify diagnosis of TMD in the routine clinical setting, the CEP-TMD provides a list of the main criteria for each diagnostic group and subgroup derived from the RDC/TMD. It is quicker and less sophisticated than the RDC/TMD and provides an instant physical diagnosis, which may make it more intuitive and attractive for general dental practitioners to use in routine clinical practice [23]. The RDC/TMD remains the gold standard for use in research [19].

In future, studies can also be done to determine if other simplified versions of the RDC/TMD's clinical examination, similar to the Clinical Examination Protocol-TMD (CEP-TMD), would have validity but be quicker to carry out than RDC/TMD.

\section{Conclusion}

Being able to diagnose TMD patients' physical problems effectively is helpful for dentists in deciding whether to treat or to refer. It also helps standardize communication during referral. More importantly, it would allow GDPs to allay patients' fears immediately rather than waiting for a specialist or hospital based consultant to do so which sometimes, due to waiting lists, takes some months. If the dentist decides to treat the patient, the examination form has the potential to provide a useful means of monitoring changes at follow-up.
The results of this survey accentuates the need for dental clinicians, worldwide, to use the standard protocols for diagnosis for TMD. This will aid in scrutinizing any suspected TMD cases and avoid negligence of the existing underlying disease.

\section{References}

[1]. Lobbezoo F, Drangsholt M, Peck C, Sato H, Kopp S, Svensson P. Topical review: new insights into the pathology and diagnosis of disorders of the temporomandibular joint. J Orofac Pain. 2004 Summer;18(3):181-91.Pubmed PMID: 15508997.

[2]. Pullinger AG, Seligman DA, Solberg WK. Temporomandibular disorders. Part II: Occlusal factors associated with temporomandibular joint tenderness and dysfunction. J Prosthet Dent. 1988 Mar;59(3):363-7. Pubmed PMID: 3162277.

[3]. Petersson A. What you can and cannot see in TMJ imaging--an overview related to the RDC/TMD diagnostic system. J Oral Rehabil. 2010 Oct;37(10):771-8. Pubmed PMID: 20492436.

[4]. Al-Ani Z, Gray R. TMD current concepts: 2. Imaging and treatment options. An update. Dent Update. 2007 Jul-Aug;34(6):356-8, 361-4, 367-70. Pubmed PMID: 17784585.

[5]. Hunter A, Kalathingal S. Diagnostic imaging for temporomandibular disorders and orofacial pain. Dent Clin North Am. 2013 Jul;57(3):405-18. Pubmed PMID: 23809300.

[6]. Murphy MK, MacBarb RF, Wong ME, Athanasiou KA. Temporomandibular disorders: a review of etiology, clinical management, and tissue engineering strategies. Int J Oral Maxillofac Implants. 2013 Nov;28(6):e393-414. PubmedPMID: 24278954.

[7]. Schiffman E, Ohrbach R, Truelove E, Look J, Anderson G, Goulet J-P, et al. Diagnostic Criteria for Temporomandibular Disorders (DC/TMD) for Clinical and Research Applications: recommendations of the International RDC/TMD Consortium Network* and Orofacial Pain Special Interest Group $\dagger$. J Oral Facial Pain Headache. 2014 Winter;28(1):6-27.Pubmed PMID: 24482784

[8]. Carlsson GE. Temporomandibular joint disorders. InFunctional Occlusion in Restorative Dentistry and Prosthodontics 2016 Jan 1 (pp. 161-171). Mosby.

[9]. Muthukrishnan A, Sekar GS. Prevalence of temporomandibular disorders in Chennai population.Journal of Indian Academy of Oral Medicine and Radiology. 2015 Oct 1;27(4):508.

[10]. Laskin DM. Report of the president's conference on the examination, diagnosis, and management of temporomandibular disorders. J Am Dent Assoc. 1983 Jan;106(1):75-7. Pubmed PMID: 6574170.

[11]. Tanaka E, Detamore MS, Mercuri LG. Degenerative disorders of the temporomandibular joint: etiology, diagnosis, and treatment. J Dent Res. 2008 Apr; 87(4):296-307. Pubmed PMID: 18362309.

[12]. Truelove E, Dworkin SF, Schubert MM, LeResche L, Sommers EE. Signs and symptoms of TMJ/MPD pain. Pain. 1984 Jan 1;18:S112.

[13]. Dolwick MF, Riggs RR. Diagnosis and treatment of internal derangements of the temporomandibular joint. Dent Clin North Am. 1983 Jul;27(3):56172. Pubmed PMID: 6578966.

[14]. Shukla D, Muthusekhar MR. Efficacy of low-level laser therapy in temporomandibular disorders: A systematic review. Natl J Maxillofac Surg. 2016 Jan-Jun;7(1):62-66. Pubmed PMID: 28163481.

[15]. Griffiths RH. Report of the president's conference on the examination, diagnosis, and management of temporomandibular disorders. J Am Dent Assoc. 1983 Jan;106(1):75-7. Pubmed PMID: 6574170. 
[16]. National Institutes of Health Technology Assessment Conference on Management of Temporomandibular Disorders. Bethesda, Maryland, April 29May 1, 1996. Proceedings. Oral Surg Oral Med Oral Pathol Oral Radiol Endod. 1997 Jan;83(1):49-183.Pubmed PMID: 9007923.

[17]. McNeil C. Temporomandibular disorders: guidelines for classifications, assessment, and management. American Academy of Orofacial Pain. $1993 . .141 \mathrm{p}$.

[18]. Look JO, Schiffman EL, Truelove EL, Ahmad M. Reliability and validity of Axis I of the Research Diagnostic Criteria for Temporomandibular Disorders (RDC/TMD) with proposed revisions. J Oral Rehabil. 2010 Oct;37(10):744-59. Pubmed PMID: 20663019.

[19]. Hasanain F, Durham J, Moufti A, Steen IN, Wassell RW. Adapting the diagnostic definitions of the RDC/TMD to routine clinical practice: a feasibility study. J Dent. 2009 Dec;37(12):955-62. Pubmed PMID: 19682534.

[20]. Tegelberg A, List T, Wahlund K, Wenneberg B. Temporomandibular disorders in children and adolescents: a survey of dentists' attitudes, routine and experience. Swed Dent J. 2001;25(3):119-27.Pubmed PMID: 11813448.

[21]. Durham J, Exley C, Wassell R, Steele JG. 'Management is a black art'--professional ideologies with respect to temporomandibular disorders. $\mathrm{Br}$ Dent J. 2007 Jun 9;202(11):E29; discussion 682-3. Pubmed PMID: 17471185.

[22]. Moufti MA. Developing an outcome measure in temporomandibular disorders [Internet]. University of Newcastle upon Tyne; 2007.

[23]. Durham J, Wassell RW. Recent Advancements in Temporomandibular Disorders (TMDs). Rev Pain. 2011 Mar;5(1):18-25. Pubmed PMID: 26527468. 